Supporting information

\title{
A multi-reporter bacterial 2-hybrid assay for the high-throughput and dynamic assay of PDZ domain - peptide interactions
}

David M. Ichikawa ${ }^{1,6}$, Carles Corbi-Verge ${ }^{2,6}$, Michael J. Shen ${ }^{1}$, Jamie Snider ${ }^{2}$, Victoria Wong' Igor Stagljar ${ }^{2,3,4}$, Philip M. Kim²,3,5,7, and Marcus B. Noyes ${ }^{1,7}$

1. Department of Biochemistry Molecular Pharmacology and Institute for Systems Genetics, NYU Langone Health, New York, NY 10016, USA.

2. Donnelly Centre for Cellular and Biomolecular Research, University of Toronto, Toronto M5S 3E1, Canada

3. Department of Molecular Genetics, University of Toronto, Ontario, Canada.

4. Department of Biochemistry, University of Toronto, Ontario, Canada.

5. Department of Computer Science, University of Toronto, Ontario, Canada

6. These authors contributed equally

7. Corresponding author

\section{Table of Contents}

Figure S1 Page 2

Figure S2 Page 3

Figure S3 Page 4

Figure S4 Page 5

Figure S5 Page 6

Figure S6 Page 7

Figure S7 Page 8

Table S1.... Page 9

Supplementary Methods. Page 10 


\section{ERBIN $\quad$ MAGI1 $\quad$ DLG4 $\quad$ SCRIB $\begin{array}{lll}\text { PDZ } & \text { PDZ2 } & \text { PDZ1 }\end{array}$}

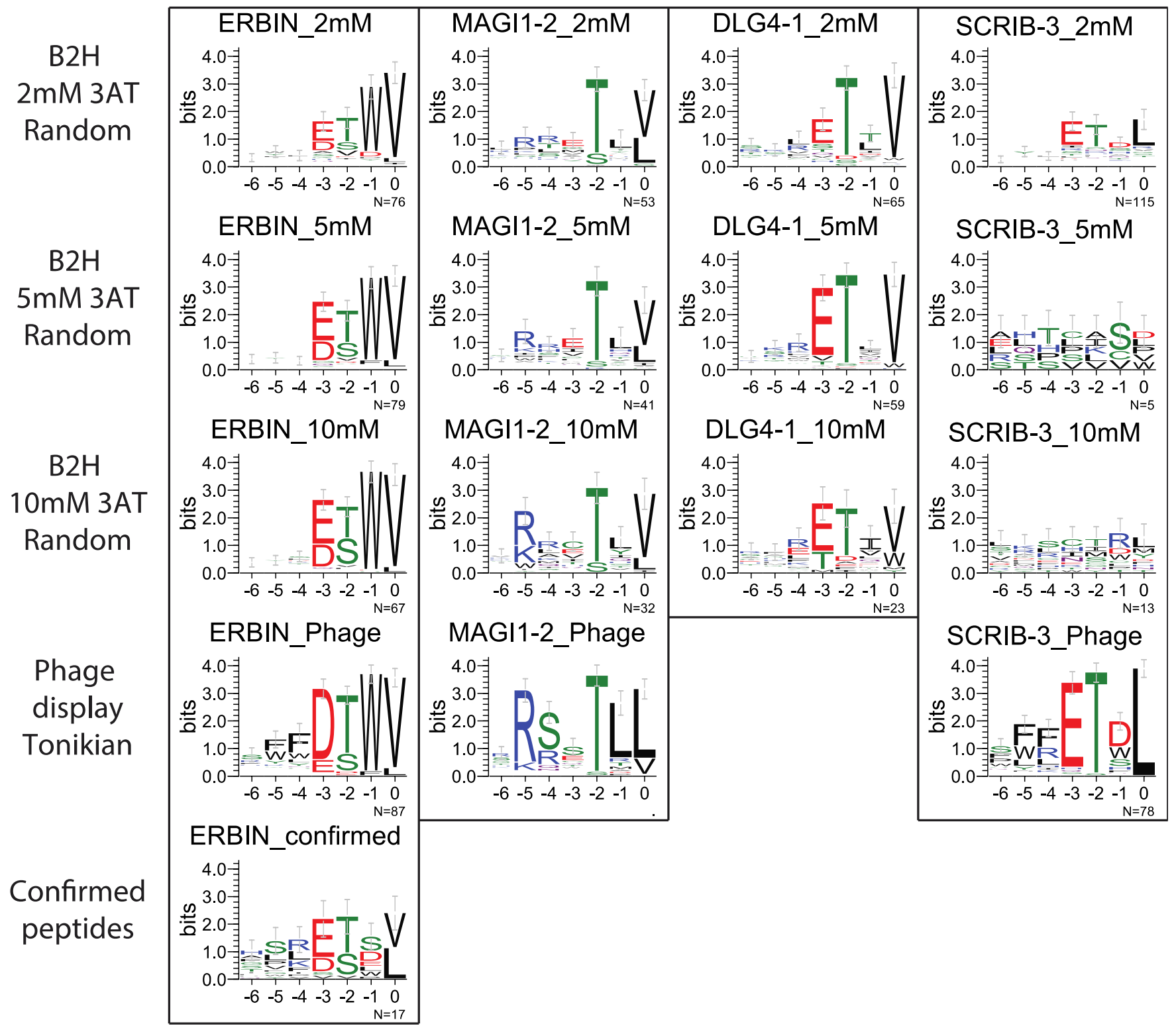

Figure S1. PDZ specificities determined by MR-B1H plate selections. Top 3 rows, Peptides were selected from the random library by the PDZ domain labeled above each motif. This PDZ domain name is followed by the amount of 3-AT present in the plate for the selection represented. Bottom rows, where applicable, motifs are shown that represent peptides selected by phage display, also using a random library ${ }^{2}$. For the Erbin PDZ, a motif of peptides known to bind the domain is also shown ${ }^{5}$. 

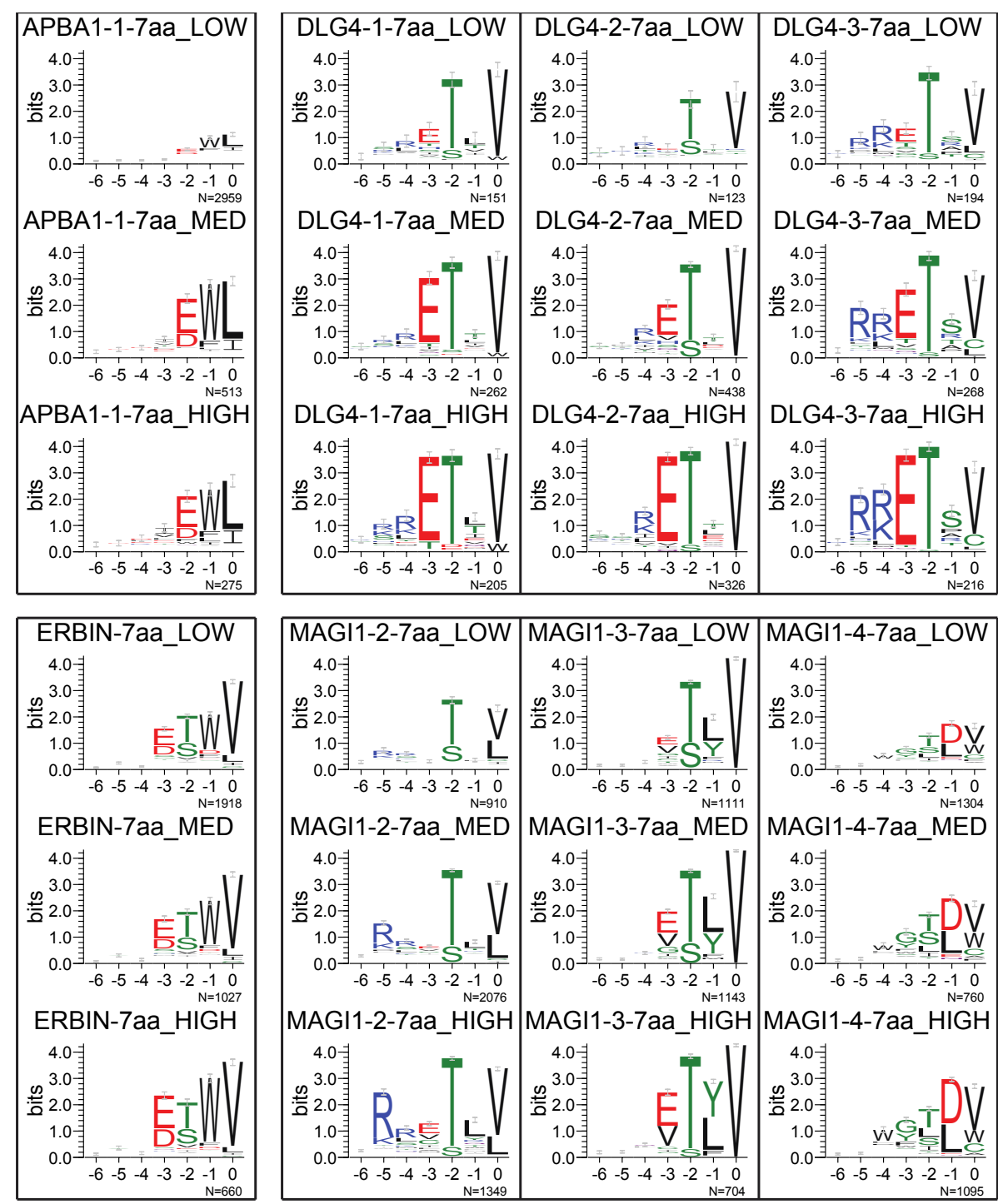

\begin{tabular}{|l|l|l|}
\hline MAGI1-2-7aa_LOW & MAGI1-3-7aa_LOW & MAGI1-4-7aa_LOW \\
\hline
\end{tabular}
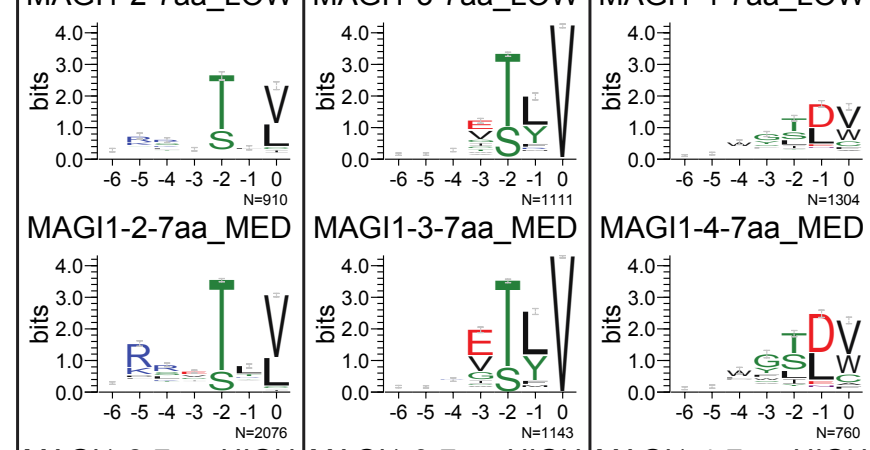

MAGI1-3-7aa_MED

MAGI1-4-7aa_MED

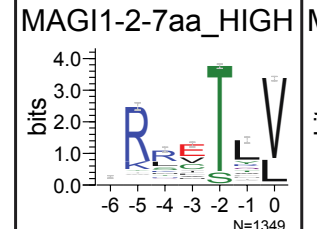

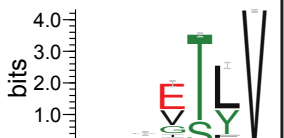
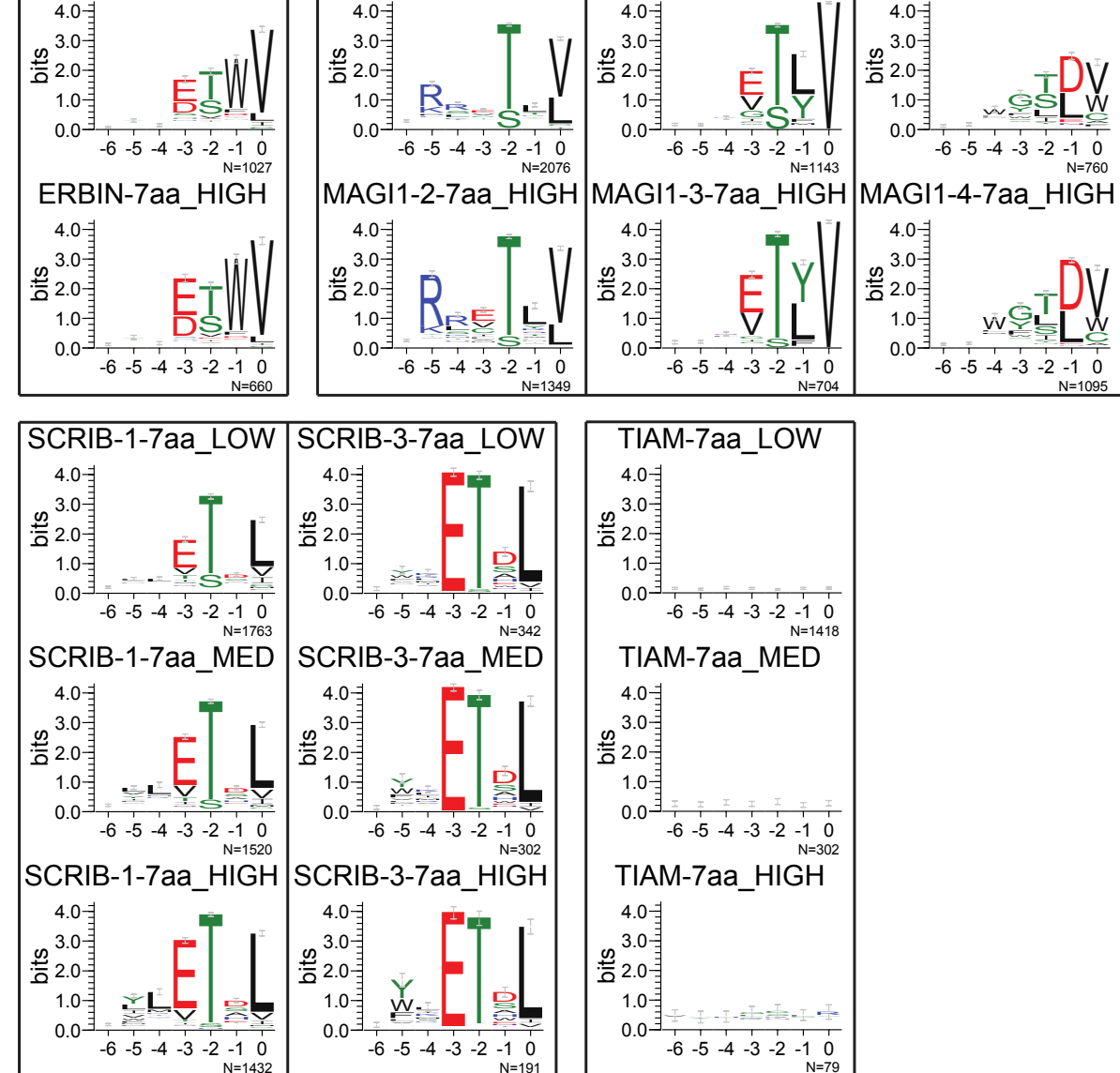

SCRIB-3-7aa_LOW

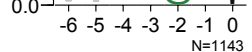

MAGI1-4-7aa_HIGH
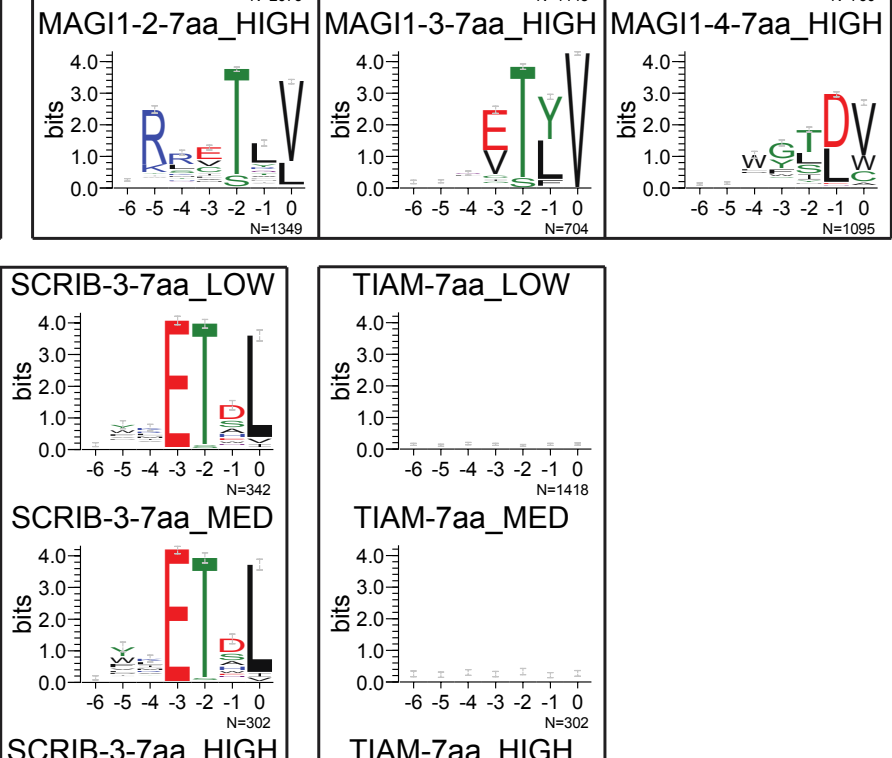

TIAM-7aa_LOW

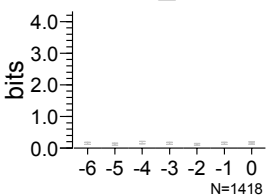

TIAM-7aa_MED
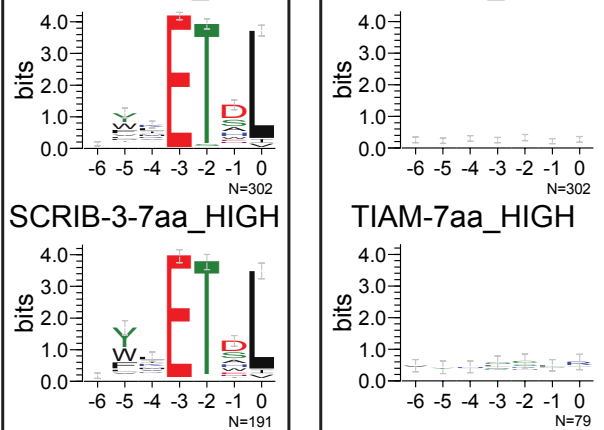

TIAM-7aa_HIGH

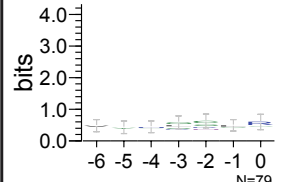

Figure S2. PDZ specificities determined by MR-B1H selection and GFP sort using a 7aa Random library. Motifs represent peptides selected from the random library and recovered from populations at various GFP levels. The name of the PDZ domain tested is labeled above each motif as well as the GFP level it represents (LOW, MED (medium), or HIGH). 

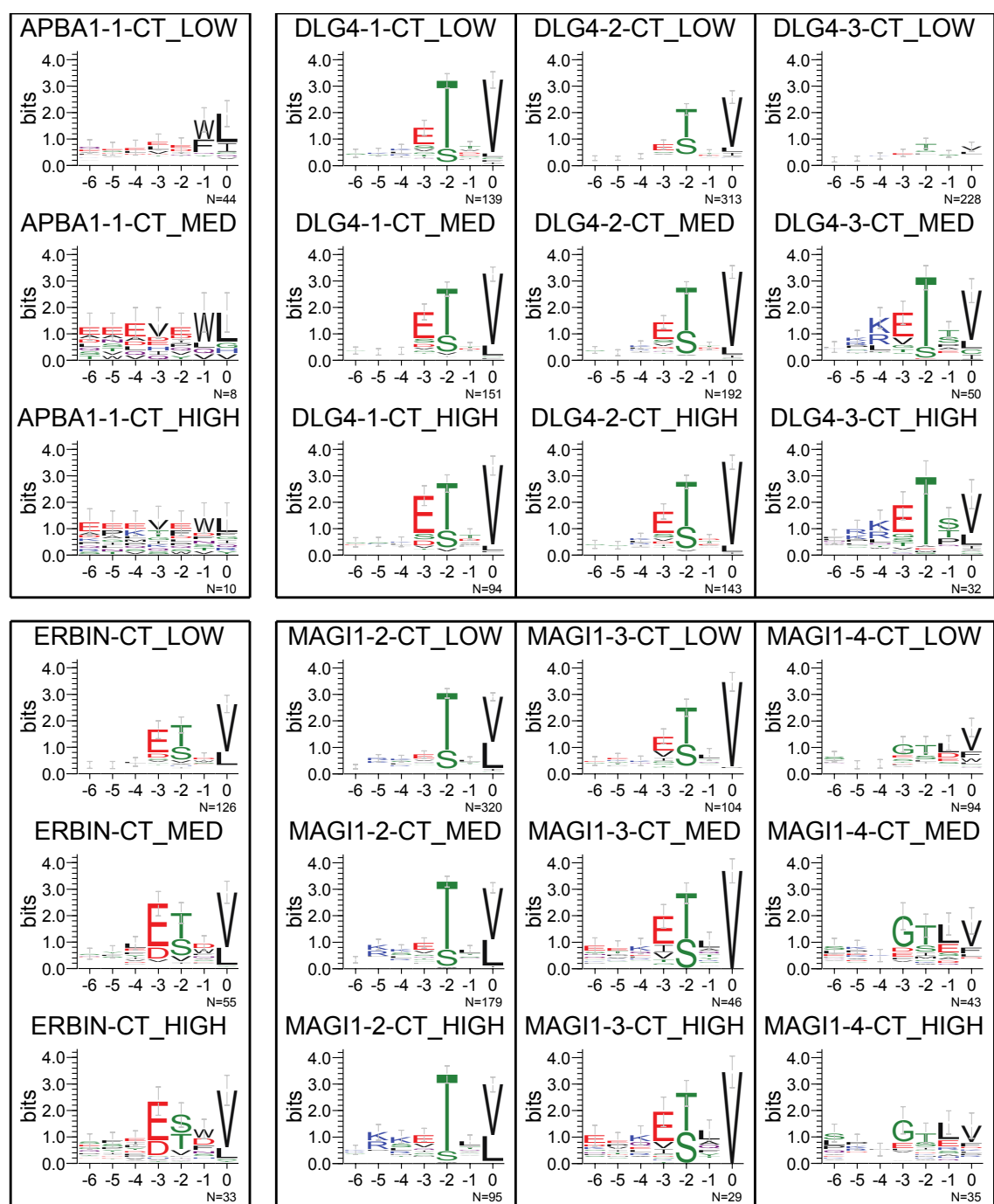

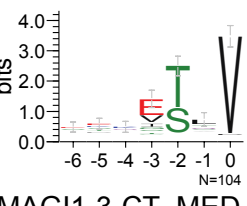

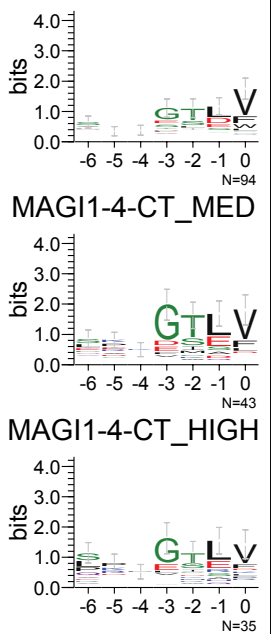

SCRIB-1-CT_LOW

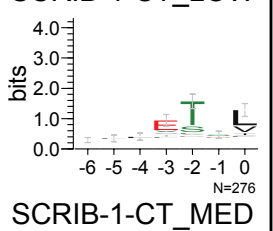

SCRIB-3-CT_LOW

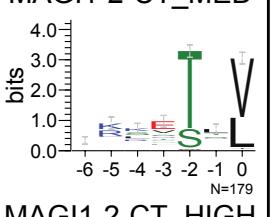

MAGI1-3-CT_MED

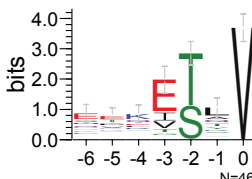

MAGI1-2-CT_HIGH

MAGI1-3-CT HIGH

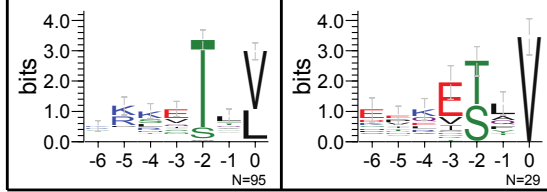

MAGI1-4-CT HIGH
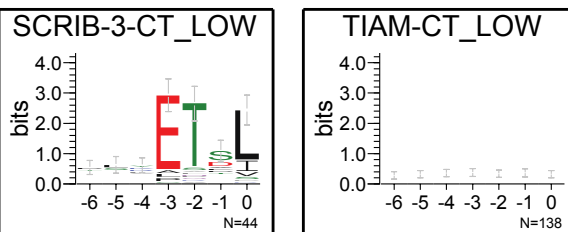

MPDZ-10-CT_LOW

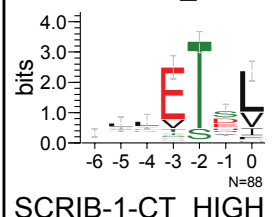

SCRIB-3-CT_MED
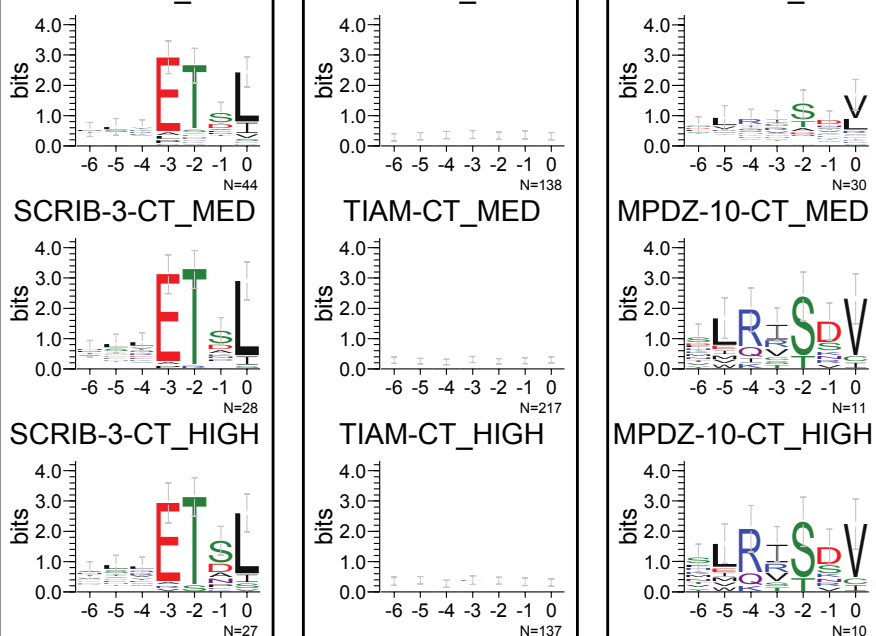

Figure S3. PDZ specificities determined by MR-B1H selection and GFP sort using a Natural library. Motifs represent peptides selected from the Natural library that consists of $\sim 50,000$ C-termini of human ORFs and splice variants ${ }^{1}$. These selected peptides were recovered from populations at various GFP levels. The name of the PDZ domain tested is labeled above each motif as well as the GFP level it represents (LOW, MED (medium), or HIGH). 
A.

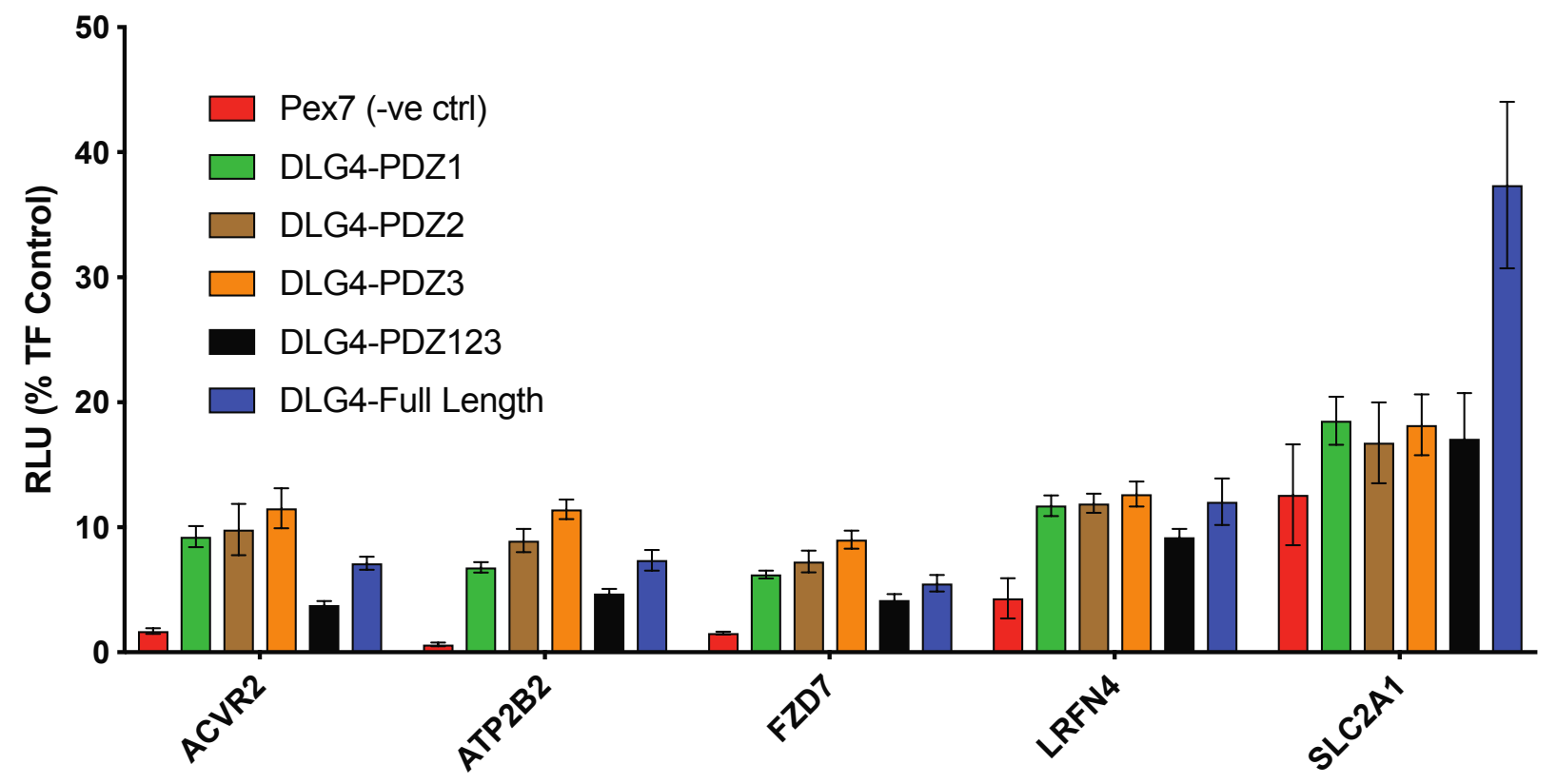

B.

\begin{tabular}{|r|c|c|c|c|c|}
\hline & $\begin{array}{c}\text { DLG4- } \\
\text { PDZ1 }\end{array}$ & $\begin{array}{c}\text { DLG4- } \\
\text { PDZ2 }\end{array}$ & $\begin{array}{c}\text { DLG4- } \\
\text { PDZ3 }\end{array}$ & $\begin{array}{c}\text { DLG4- } \\
\text { PDZ123 }\end{array}$ & $\begin{array}{c}\text { DLG4-Full- } \\
\text { Length }\end{array}$ \\
\hline ACVR2B & +++ & +++ & +++ & - & +++ \\
\hline ATP2B2 & +++ & +++ & +++ & + & +++ \\
\hline FZD7 & + & +++ & +++ & - & + \\
\hline LRFN4 & +++ & +++ & +++ & + & +++ \\
\hline SLC2A1 & +++ & + & ++ & + & +++ \\
\hline
\end{tabular}

Figure S4. Validation of full-length PDZ interactions by MaMTH assay. Full-length DLG4 and truncated versions that include each or all of the PDZ domains were tested for their ability to bind full-length versions of predicted binding partners. A) The control construct (Pex7) or the DLG4 variants were paired with the predicted full-length partners noted on the X-axis. For each pairing, luciferase expression was measured as Relative Light Units (RLU) and normalized as the fraction of the output produced by a control transcription factor. B) A chart of $p$-values obtained from a 2-way ANOVA (multiple comparisons) between the indicated interaction and the corresponding Pex7 negative control $(p \geq 0.05(-) ; p<0.05(+) ; p<0.005(++) ; p<0.0005(+++))$. 


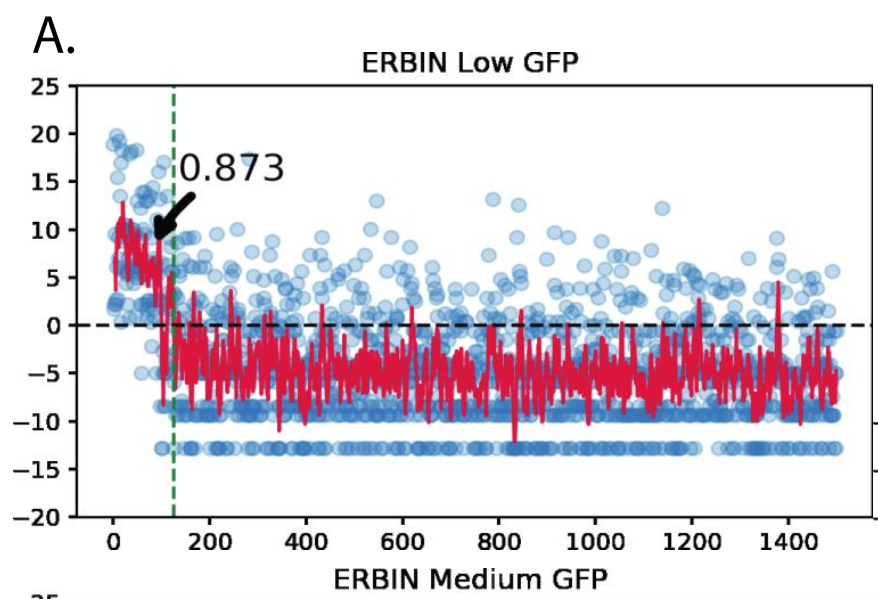

B.
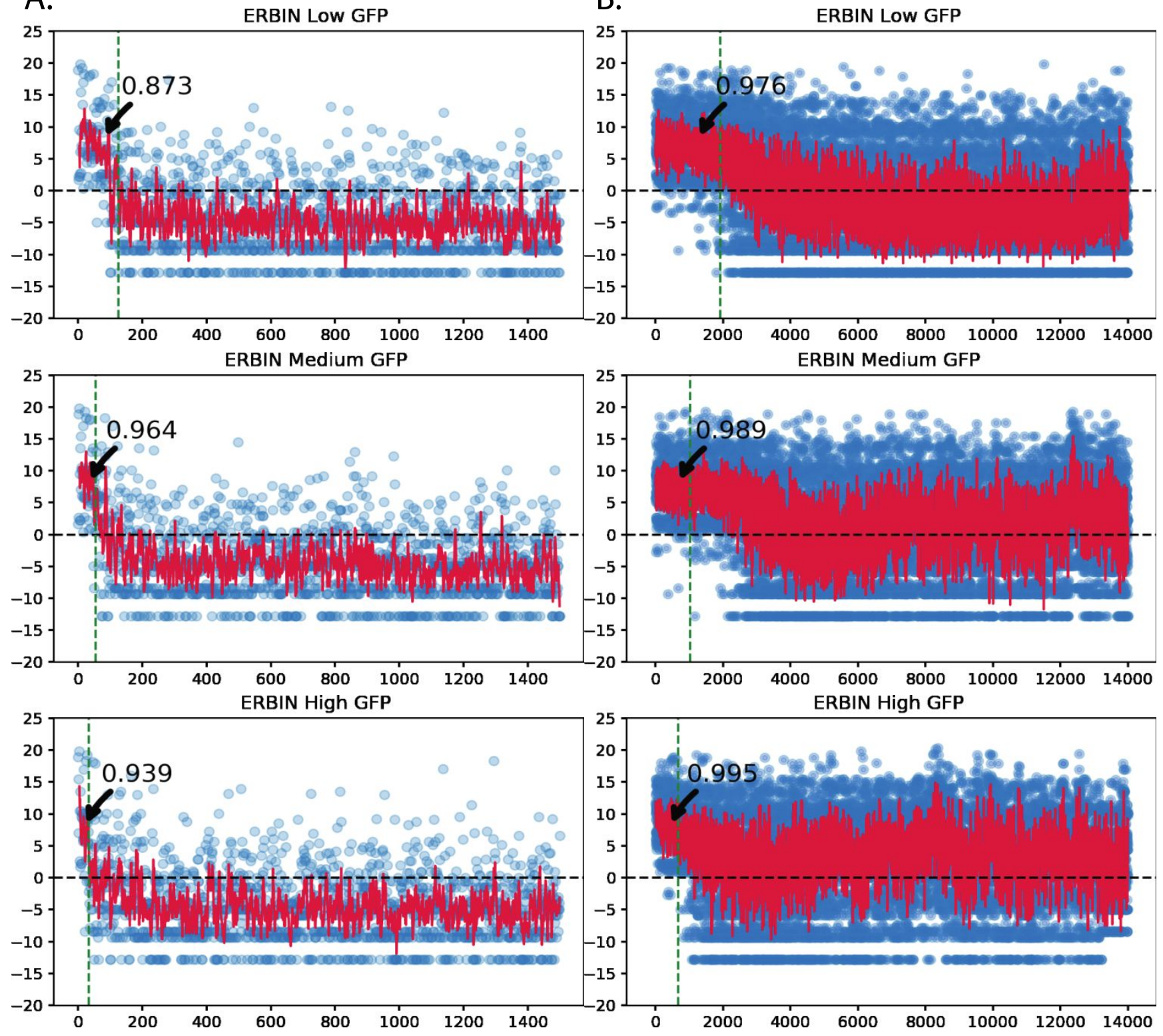

Figure S5. Peptides ranked by read number (X-axis) and scored using a PWM built from a set of confirmed peptides (Y-axis) for the ERBIN PDZ domain. A) Peptide scores from the Natural library (cut-off $=3.4$ normalized read counts) are shown. B) Peptide scores from the Random library (cut-off $=$ top 5\%) are shown. The dashed green line marks the cut-off threshold, and the red line is the moving average for the PWM score. The fraction of peptides a one the cut-off with a positive PWM score is reported in each panel to provide a coarse estimation of the false positive rate. 
A.

pB1H2 UV5 Zif268-Random library

CCATGGGTGCGGCCGCGGACTACAAGGATGACGACGACAAGTTCCGGACGGGCTCCAAGACACCGCCT

CACGGTACCGAGCGCCCATATGCTTGCCCTGTCGAGTCCTGCGATCGCCGCTTTTCTCGCTCGGATGAG

CTTACCCGCCATATCCGCATCCATACCGGTCAGAAGCCCTTCCAGTGTCGAATCTGCATGCGTAACTTCA

GTCGTAGTGACCACCTTACCACCCACATCCGCACCCACACAGGCGAGAAGCCTTTTTGCCTGTGACATTT

GTGGGAGGAAGTTTGCCAGGAGTGATGAACGCAAGAGGCATACCAAAATCCATCTGCGCGGATCCGGC

GGTGGGAGCGGAGGCNNSNNSNNSNNSNNSNNSNNSTAATAGGTCTAGA

Restriction sites (Ncol (encodes start codon) and Xbal)

FLAG-tag

Zif268 zinc fingers

Linker

Library

B.

pB1H2 UV5 Zif268-Natural library

CCATGGGTGCGGCCGCGGACTACAAGGATGACGACGACAAGTTCCGGACGGGCTCCAAGACACCGCCT CACGGAACCGAGCGCCCATATGCTTGCCCTGTCGAGTCCTGCGATCGCCGCTTTTCTCGCTCGGATGAG

CTTACCCGCCATATCCGCATCCATACCGGTCAGAAGCCCTTCCAGTGTCGAATCTGCATGCGTAACTTCA

GTCGTAGTGACCACCTTACCACCCACATCCGCACCCACACAGGCGAGAAGCCTTTTGCCTGTGACATTT

GTGGGAGGAAGTTTGCCAGGAGTGATGAACGCAAGAGGCATACCAAAATCCATCTGCGCGGTACCGGC

GCCCCCGGTGGCGGANNNNNNNNNNNNNNNNNNNNNTGATAAACCGATACAATAAAGGCTCTAGA

Restriction sites (Kpnl and Xbal)

FLAG-tag

Zif268 zinc fingers

Linker

Library

Figure S6. Coding scheme for libraries employed. A) Zif268-Random library sequence is shown where NNS represents the code for each codon. B) Zif268-Natural library sequence is shown where NNN purely represents diversity but as these $\sim 50,000$ are natural peptides, NNN is NOT meant to indicate every possible combination. 
A.

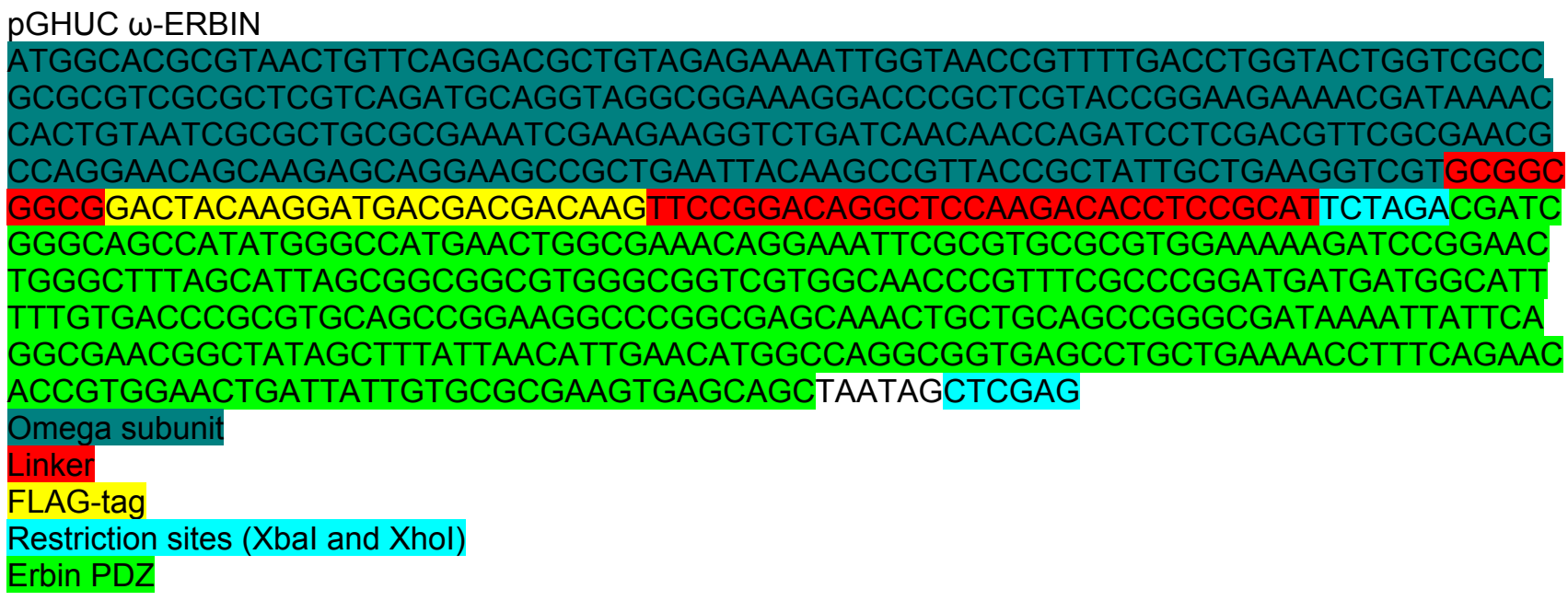

C.

pB1H2 UV5 Zif268-cons

CCATGGGTGCGGCCGCGGACTACAAGGATGACGACGACAAGTTCCGGACGGGCTCCAAGACACCGCCT CACGGTACCGAGCGCCCATATGCTTGCCCTGTCGAGTCCTGCGATCGCCGCTTTTCTCGCTCGGATGAG CTTACCCGCCATATCCGCATCCATACCGGTCAGAAGCCCTTCCAGTGTCGAATCTGCATGCGTAACTTCA GTCGTAGTGACCACCTTACCACCCACATCCGCACCCACACAGGCGAGAAGCCTTTTGCCTGTGACATTT GTGGGAGGAAGTTTGCCAGGAGTGATGAACGCAAGAGGCATACCAAAATCCATCTGCGCGGATCCGGC GGTGGGAGCGGAGGCACCGGCTGGGAAACCTGGGTGTAATAGGTCTAGA

Restriction sites ( $\mathrm{Ncol}$ (encodes start codon), BamHI (part of linker), and Xbal)

FLAG-tag

Zif268 zinc fingers

Linker

TGWETWV positive control peptide (when paired with $\omega$-ERBIN)

Figure S7. Coding scheme for the ERBIN PDZ and two controls. A) w-ERBIN PDZ sequence is shown. B) The negative control Zif268-5ala sequence is shown. C) The positive control Zif268cons is shown. The consensus TGWETWV peptide sequence has been shown to bind strongly to the ERBIN PDZ domain. 
Supplemental Table 1

\begin{tabular}{|c|c|c|c|c|c|c|c|c|}
\hline uniprot & $\begin{array}{c}\text { gene } \\
\text { name }\end{array}$ & Sequence & $\begin{array}{l}\text { Affinity } \\
(\mu \mathrm{M})\end{array}$ & DOMAIN & pubmed & $\begin{array}{c}\text { NR LOW } \\
\text { GFP }\end{array}$ & $\begin{array}{c}\text { NR MED } \\
\text { GFP }\end{array}$ & $\begin{array}{l}\text { NR HIGH } \\
\text { GFP }\end{array}$ \\
\hline Q12879 & GRIN2A & PSIESDV & 5.25 & $\begin{array}{l}\text { DLG4- } \\
\text { PDZ1 }\end{array}$ & 11937501 & 76.56 & 122.12 & 53.23 \\
\hline P22459 & KCNA4 & KAVETDV & 2.23 & $\begin{array}{l}\text { DLG4- } \\
\text { PDZ1 }\end{array}$ & 11937501 & 82.01 & 204.13 & 315.91 \\
\hline Q13224 & GRIN2B & SSIESDV & 2.3 & $\begin{array}{l}\text { DLG4- } \\
\text { PDZ1 }\end{array}$ & 11937501 & 72.66 & 132.57 & 53.10 \\
\hline O15399 & GRIN2D & SSLESEV & 2.87 & $\begin{array}{l}\text { DLG4- } \\
\text { PDZ1 }\end{array}$ & 11937501 & 25.50 & 33.03 & 22.73 \\
\hline Q09470 & KCNA1 & SKLLTDV & 20.79 & $\begin{array}{l}\text { DLG4- } \\
\text { PDZ1 }\end{array}$ & 11937501 & 4.32 & 1.57 & 0.02 \\
\hline Q9P021 & CRIPT & NYKQTSV & 20.09 & $\begin{array}{l}\text { DLG4- } \\
\text { PDZ1 }\end{array}$ & 11937501 & 33.78 & 0.90 & 0.19 \\
\hline Q12879 & GRIN2A & PSIESDV & 0.75 & $\begin{array}{l}\text { DLG4- } \\
\text { PDZ2 }\end{array}$ & 11937501 & 20.29 & 126.82 & 287.75 \\
\hline Q13224 & GRIN2B & SSIESDV & 0.91 & $\begin{array}{l}\text { DLG4- } \\
\text { PDZ2 }\end{array}$ & 11937501 & 37.58 & 115.82 & 325.07 \\
\hline P22459 & KCNA4 & KAVETDV & 0.75 & $\begin{array}{l}\text { DLG4- } \\
\text { PDZ2 }\end{array}$ & 11937501 & 34.52 & 110.24 & 460.78 \\
\hline 015399 & GRIN2D & SSLESEV & 0.6 & $\begin{array}{l}\text { DLG4- } \\
\text { PDZ2 }\end{array}$ & 11937501 & 18.78 & 54.46 & 164.41 \\
\hline Q09470 & KCNA1 & SKLLTDV & 6.7 & $\begin{array}{l}\text { DLG4- } \\
\text { PDZ2 }\end{array}$ & 11937501 & 28.60 & 4.84 & 1.99 \\
\hline Q9P021 & CRIPT & NYKQTSV & 1.2 & $\begin{array}{l}\text { DLG4- } \\
\text { PDZ2 }\end{array}$ & 11937501 & 14.55 & 6.17 & 2.33 \\
\hline Q9P021 & CRIPT & NYKQTSV & 2.1 & $\begin{array}{l}\text { DLG4- } \\
\text { PDZ3 }\end{array}$ & 17474715 & 63.88 & 237.48 & 77.91 \\
\hline O00192 & ARVCF & QPVDSWV & 8 & $\begin{array}{l}\text { Erbin- } \\
\text { PDZ }\end{array}$ & 15465056 & 754.32 & $1,065.05$ & $4,282.18$ \\
\hline P25054 & APC & SYLVTSV & 19.2 & $\begin{array}{l}\text { Erbin- } \\
\text { PDZ }\end{array}$ & 15465056 & 125.30 & 98.84 & 12.97 \\
\hline Q9UQB3 & CTNND2 & ASPDSWV & 37 & $\begin{array}{l}\text { Erbin- } \\
\text { PDZ }\end{array}$ & 11821434 & 195.93 & 737.37 & 754.06 \\
\hline Q13224 & GRIN2B & SSIESDV & 57.1 & $\begin{array}{l}\text { Erbin- } \\
\text { PDZ }\end{array}$ & 15465056 & 142.48 & 68.87 & 4.66 \\
\hline P35222 & CTNNB1 & AWFDTDL & 53.1 & $\begin{array}{l}\text { Erbin- } \\
\text { PDZ }\end{array}$ & 15465056 & 40.16 & 10.66 & 1.79 \\
\hline P27037 & ACVR2A & PPKESSL & 173.7 & $\begin{array}{l}\text { Erbin- } \\
\text { PDZ }\end{array}$ & 15465056 & 4.12 & 1.12 & 0.10 \\
\hline P11274 & BCR & ILFSTEV & 41 & $\begin{array}{l}\text { Erbin- } \\
\text { PDZ }\end{array}$ & 15465056 & 4.75 & 0.00 & 0.55 \\
\hline P33402 & $\begin{array}{c}\text { GUCY1A } \\
2\end{array}$ & FLRETSL & 10 & $\begin{array}{c}\text { Scribble- } \\
\text { PDZ3 }\end{array}$ & 24550280 & 407.14 & 305.09 & 164.90 \\
\hline $\begin{array}{c}\text { A0A087W } \\
\text { ZD4 }\end{array}$ & WDR98 & RFLETKL & 5.8 & $\begin{array}{c}\text { Scribble- } \\
\text { PDZ3 }\end{array}$ & 24550280 & 33.66 & 14.86 & 12.24 \\
\hline Q14155 & $\begin{array}{c}\text { ARHGEF } \\
7\end{array}$ & AWDETNL & 3.5 & $\begin{array}{c}\text { Scribble- } \\
\text { PDZ3 }\end{array}$ & 24550280 & 6.41 & 5.60 & 8.02 \\
\hline P53778 & MAPK12 & VSKETPL & 5 & $\begin{array}{l}\text { Scribble- } \\
\text { PDZ3 }\end{array}$ & 24550280 & 6.47 & 4.75 & 5.72 \\
\hline Q14155 & $\begin{array}{c}\text { ARHGEF } \\
7\end{array}$ & AWDETNL & 2.3 & $\begin{array}{c}\text { Scribble- } \\
\text { PDZ1 }\end{array}$ & 24550280 & 106.93 & 260.73 & 148.11 \\
\hline $\begin{array}{c}\text { A0A087W } \\
\text { ZD4 }\end{array}$ & WDR98 & RFLETKL & 2.1 & $\begin{array}{c}\text { Scribble- } \\
\text { PDZ1 }\end{array}$ & 24550280 & 209.40 & 350.38 & $1,526.30$ \\
\hline
\end{tabular}

**NR $=$ Normalized Reads 


\section{Methods}

\section{Library and plasmid construction}

The Random library was constructed as previously described ${ }^{3,4}$. In short, 96 separate 15 -cycle PCR reactions were performed using Zif268 as a template. The 3' oligonucleotide includes the code for the linker and random peptide region, stop codons and an Xbal site. In addition, the randomization scheme was requested to be hand mixed by IDT as our prior work had shown this approach led to a more true randomization (unpublished data). The low cycle number reduces the amplification of polymerase error and separate reactions minimizes the influence that any bias from a single reaction will have on the entire population when 96 reactions are pooled. The forward primer contained the Ncol restriction site that includes the start codon followed by an N-terminal flag tag. The pooled PCR product was digested with $\mathrm{Ncol}$ and Xbal and cloned into our expression vector that utilizes a LacUV5 promoter for expression (Figure S6).

The Natural library was constructed similarly, except the amplification only included the peptide region. Oligonucleotides were designed to amplify around the peptide region on the phagemid library previously investigated ${ }^{1}$. On the end of this sequence were placed cloning sites Kpnl and Xbal (Figure S6).

Omega-PDZ fusions were created by restriction cloning into the $\mathrm{pGHUC}^{6}$ vector where the omega-PDZ constructs are expressed from a lacUV5 promoter. PDZ domains can easily be cloned between Xbal and Xhol site to result in the omega fusions (Figure S7). PDZ domains were purchased commercially as dsDNA G-blocks from IDT.

${ }^{*}$ All base plasmids and complete sequences will be made available at addgene.

\section{Selections on plates}

One $\mu \mathrm{g}$ of prey (zif268-peptide library fusion) was transformed with $1 \mu \mathrm{g}$ of concentrated bait ( $\omega$ PDZ fusion) reporter into the selection strain (E. coli - strain genotype: USO $\Delta$ rpoZ, $\Delta$ hisB, $\Delta$ pyrF $)^{7}$. Cells were recovered at $37^{\circ} \mathrm{C}$ first in $10 \mathrm{~mL} \mathrm{SOC}$ for 1 hour, and then in complete NM media for another hour, before washing with NM media that lacks histidine and concentrating to $1 \mathrm{~mL}$ for overnight storage. The washed cells are titrated by 10 -fold serial dilutions on rich media plates supplemented with appropriate antibiotics to determine total number of transformants that took up both bait and prey plasmids (Kanamycin ${ }^{r}$ and Ampicillin', respectively). The following day, $5 \mathrm{e} 7$ cells were plated on $15 \mathrm{~mm} X 150 \mathrm{~mm}$ NM media plates (-histidine) containing 2, 5, or $10 \mathrm{mM} 3$ Amino-1,2,4-triazole (3AT) and grown for 36 to 48 hours at $37^{\circ} \mathrm{C}$. $5 \mathrm{e} 7$ cells are used to avoid crowding on the plates and we have not found that this severely limits the number of colonies that survive. However, if a PDZ domain were very specific, it may require the investigation of larger fractions of the library to recover a substantial number of variants it is able to interact with. In this case we would plate $1 \mathrm{e} 9$ on $245 \mathrm{~mm}$ X $245 \mathrm{~mm}$ ("large") plates as we have done for other applications including the selections for sorting below (1e9 plated on 1mM 3AT for each PDZ to be sorted). Colony PCR was performed on surviving colonies and the sequences were quality checked and compiled into sequence logos using the WebLogo tool (http://weblogo.berkeley.edu/logo.cgi.

\section{Selection in liquid culture}


For liquid selection and sorting, $\sim 1 \mathrm{e} 9$ cells were plated on large plates above at low stringency as detailed above. The surviving colonies per pooled and DNA harvested. This "round 1 DNA" was retransformed with the PDZ reporter under investigation, and treated just as a plate selection through the titration step. At this point, when transformants can be counted, $10^{6}$ cells are inoculated into $100 \mathrm{~mL}$ NM media (-histidine) with $1 \mathrm{mM} 3 \mathrm{AT}$ and grown in shaking incubators at $37^{\circ} \mathrm{C}$. We have found that the most informative data can be found just as the GFP expression approaches its maximum. However, it can be challenging to determine when that time point will be. Therefore, for each PDZ $\sim \mathrm{mL}$ of culture was spun down and resuspended in cold PBS + $0.5 \%$ FBS every 3 hours starting 12 hours after inoculation and ending 48 hours after inoculation. Samples were run on a SONY SH800 cell cytometer and the mean GFP was recorded for each time point. Populations of GFP positive cells (low, medium, and high) just prior to saturation were recovered as well as a population of cells that were not expressing GFP (negatives). 1e6 cells from the four sub-populations of GFP were sorted: negative, low, medium, and high, with the mean GFP of each population $\sim 5$ fold higher than the preceding one. As noted, the time-point just prior to GFP saturation was chosen for sorting. $~ 5 \mathrm{e} 5$ cells/GFP population were plated on 2XYT agar with antibiotics and grown to single colony resolution ( 10hours), whereupon cells were pooled, and libraries were extracted, barcoded, and sent for next-generation sequencing (NGS). In parallel, $\sim 24$ individual colonies were Sanger sequenced to compare to the NGS data.

\section{Next-generation Sequencing data processing}

After demultiplexing, all reads with an average quality Phred score of $>30$ and mapping to the C-terminal library were selected and frequencies were calculated. Random library peptides represented by multiple nucleotide sequences were selected to distinguish peptides selected at the protein level (codon diversity but the same peptide) from background, from breakthrough colonies (these would be a single DNA sequence as their breakthrough would have nothing to do with peptide enrichment). Reads not matching the selection criteria were marked as undetermined and discarded. Finally, the reads were then normalized to the total number of peptides read for that GFP population, and a scalar factor was applied for readability purposes.

To select the set of peptides that interact with the PDZ domains of the Natural library screenings, we required a minimum threshold of the normalized read count. In order to assign a cut-off value that provides the best separation between specific and non-specific values, we compared the peptides' ranks based on reads to PWM scoring derived from a set of biophysical validated peptides for each PDZ domain (Figure S5a). A consensus threshold of 3.4 normalized reads was applied to all the samples in order to maximize recall. The selected peptides include the majority of peptides with a high score in the PWM and the majority of the total reads for the sample. This model only seems to miss the optimal separation boundary in the low GFP level of SCRIB PDZ1.

The same approach does not wholly apply to the Random library (Figure S5b), mainly because of the size and diversity of the data collected. Consequently, it is challenging to find a consensus and simple threshold that generalizes for all the PDZ domains and samples. Here, to select the set of peptides from the random library that interact with the PDZ, we instead selected the top $5 \%$ of the peptides. This small group of peptides captures the majority of the reads (ranging from $65 \%$ to $85 \%$ percent of the total). This bimodal distribution of reads seems to be sufficient to provide a good split between non-specific binders and specific binders for all assayed samples.

The position weight matrices (PWMs) scores are based on the log-likelihood of the frequency of an amino acid $i$ at position $j$. Therefore, peptides containing highly frequent amino acids in 
conserved positions display positive scores. Negative scores indicate very rare or unobserved amino acids in the sequence of the peptide. The absence of key amino acids is a clear signal of the lack of the canonical binding motif. Consequently, one may label those peptides with negative scores within the top-ranked peptides as "false positives" and discard them from the pool of selected peptides. On the other hand, this assumption provides a very coarse estimation of the false positive rate (Figure S5), and this value cannot be considered a true estimation of the false positive rate. It may however be used as a relative comparison between the different GFP levels. In accordance with this, the increase in the recall of reported partners in the low GFP levels is not translated to a dramatic increase in the false positive rate detected among the top ranked peptides.

\section{MaMTH validation assays}

Entry clones were prepared in vector pDONR223, and moved into either MaMTH C-tagging bait Destination vector A1345 (for ACVR2B, ATP2B2, FZD7, LRFN4, and SLC2A1) or MaMTH Ntagging prey Destination vector A1245 (for DLG4, PEX7 and all PDZ constructs) using the Gateway Cloning Technology (Thermo Fisher Scientific). MaMTH assays were carried out as previously described ${ }^{8}$. In brief, HEK293 MaMTH reporter cells were seeded in 96-well plates and grown in DMEM (containing 10\%FBS and $1 \%$ Penicillin-Streptomycin) overnight at $37^{\circ} \mathrm{C} / 5 \%$ $\mathrm{CO}_{2}$. Cells were co-transfected with bait and prey plasmid constructs using X-tremeGENE 9 (Roche) as per manufacturer instructions. Five hours after transfection, media containing transfection reagent was removed and replaced with fresh DMEM/10\%FBS/1\%PS containing $0.5 \mu \mathrm{g} / \mathrm{mL}$ tetracycline to induce bait expression. After 24 hours growth at $37^{\circ} \mathrm{C} / 5 \% \mathrm{CO}_{2}$ luciferase activity was measured by chemiluminescence using a Clariostar microplate reader (BMG Labtech).

1 Ivarsson, Y. et al. Large-scale interaction profiling of PDZ domains through proteomic peptidephage display using human and viral phage peptidomes. Proc Natl Acad Sci US A 111, 25422547, doi:10.1073/pnas.1312296111 (2014).

2 Tonikian, R. et al. A specificity map for the PDZ domain family. PLoS Biol6, e239, doi:10.1371/journal.pbio.0060239 (2008).

3 Persikov, A. V., Rowland, E. F., Oakes, B. L., Singh, M. \& Noyes, M. B. Deep sequencing of large library selections allows computational discovery of diverse sets of zinc fingers that bind common targets. Nucleic Acids Res 42, 1497-1508, doi:10.1093/nar/gkt1034 (2014).

4 Persikov, A. V. et al. A systematic survey of the Cys2His2 zinc finger DNA-binding landscape. Nucleic Acids Res 43, 1965-1984, doi:10.1093/nar/gku1395 (2015).

5 Calderone, A., Castagnoli, L. \& Cesareni, G. mentha: a resource for browsing integrated proteininteraction networks. Nat Methods 10, 690-691, doi:10.1038/nmeth.2561 (2013).

6 Oakes, B. L. et al. Multi-reporter selection for the design of active and more specific zinc-finger nucleases for genome editing. Nat Commun 7, 10194, doi:10.1038/ncomms10194 (2016).

7 Noyes, M. B. et al. A systematic characterization of factors that regulate Drosophila segmentation via a bacterial one-hybrid system. Nucleic Acids Res 36, 2547-2560, doi:10.1093/nar/gkn048 (2008).

8 Petschnigg, J. et al. The mammalian-membrane two-hybrid assay (MaMTH) for probing membrane-protein interactions in human cells. Nat Methods 11, 585-592, doi:10.1038/nmeth.2895 (2014). 